\title{
Fauna de Euglossina (Hymenoptera: Apidae) da Amazônia Sul-Ocidental, Acre, Brasil
}

\author{
Danielle STORCK-TONON ${ }^{1}$, Elder Ferreira MORATO², Marcio Luiz OLIVEIRA ${ }^{3}$
}

\section{RESUMO}

Machos de abelhas Euglossina foram coletados entre dezembro de 2005 e setembro de 2006 em 11 áreas florestais de diferentes tamanhos na região de Rio Branco, Acre, Amazônia Sul-Ocidental. As abelhas foram atraídas por 6 substâncias odoríferas e coletadas com rede entomológica e armadilhas. Um total de 3.675 machos de Euglossina pertencentes a 4 gêneros e 36 espécies foi coletado. Eulaema cingulata (Fabricius) foi a espécie mais comum (24,6\%), seguida por Eulaema meriana (Olivier) (14,6\%), Euglossa amazonica Dressler (10,5\%), Eulaema nigrita Lepeletier (10,5\%) e Eulaema pseudocingulata (Oliveira) (7,2\%). Cineol foi a substância que atraiu maior número de indivíduos $(23,8 \%)$ e metil salicilato o maior número de espécies $(28)$ para ambos os métodos de coleta. Foram coletados 31 indivíduos pertencentes a 9 espécies portando polinários. O número acumulado de espécies coletadas na região estabilizou a partir da $48^{\mathrm{a}}$ coleta. Poucas espécies foram abundantes, a maioria representada por menos que 50 indivíduos. A falta de um protocolo amostral padronizado tem limitado comparaçôes entre trabalhos realizados em diferentes regiões. Contudo, os resultados aqui apresentados indicam que o Acre apresenta elevada riqueza dessas abelhas.

PALAVRAS-CHAVE: Abelhas, Acre, diversidade

\section{Fauna of Euglossina (Hymenoptera: Apidae) from Southwestern Amazonia, Acre, Brazil}

\section{ABSTRACT}

Male orchid bees were collected between December 2005 and September 2006 in 11 forest areas of different sizes in the region of Rio Branco, Acre, Southwestern Amazonia, Brazil. The bees were attracted by 6 aromatic compounds and collected by insect nets and scent baited traps. A total of 3,675 males of Euglossina in 4 genera and 36 species were collected. Eulaema cingulata (Fabricius) was the most common (24.6\%), followed by Eulaema meriana (Olivier) (14.6\%), Euglossa amazonica Dressler (10.5\%), Eulaema nigrita Lepeletier (10.5\%) and Eulaema pseudocingulata (Oliveira) (7.2\%). Cineole was the scent that attracted the greatest number of individuals (23.8\%) and methyl salicylate the greatest number of species (28) for both methods of sampling. Thirty one bees of 9 species with pollinar orchid attached to their bodies were collected. The accumulative number of species stabilized after the $48^{\text {th }}$ collection. Few species were abundant; the great majority were represented by less than 50 bees. The lack of standardized sample protocols limited very much the conclusions derived from comparisons among the majority of studies on Euglossina assemblages. However, the results presented here suggest that the State of Acre is very rich in those bees compared to other regions.

KEY WORDS: Acre, Bees, diversity

\footnotetext{
1 Instituto Nacional de Pesquisas da Amazônia - Coordenação de Pesquisa em Entomologia E-mail: danistorck@inpa.gov.br ; danistorck@yahoo.com.br

2 Universidade Federal do Acre - Departamento de Ciências da Natureza. E-mail: elderfmorato@yahoo.com.br

${ }^{3}$ Instituto Nacional de Pesquisas da Amazônia - Coordenação de Pesquisa em Entomologia E-mail: mlolivei@inpa.gov.br
} 


\section{INTRODUÇÃO}

As abelhas pertencentes à sub-tribo Euglossina são conhecidas como abelhas das orquídeas. São insetos de vôo rápido, coloração fortemente metálica e abrangem cerca de 200 espécies descritas e distribuídas em cinco gêneros na região Neotropical (Roubik e Hanson, 2004).

Os gêneros Eulaema Lepeletier, 1841, Euglossa Latreille, 1802, Eufriesea Cockerell, 1909 e Exaerete Hoffmannsegg, 1817 possuem distribuição geográfica semelhante, ocorrendo desde o norte do México até o Paraguai e Argentina. O gênero Aglae Lepeletier \& Serville, 1825 ocorre na região amazônica, Guiana, Panamá, oeste da Colômbia e no Estado do Mato Grosso (Dressler, 1982; Anjos-Silva et al., 2006). Porém, existem registros de Eulaema e Euglossa nos Estados do Arizona e Florida, Estados Unidos (Minckley e Reyes, 1996; Skov e Wiley, 2005) fatos que aumentam a distribuição geográfica conhecida dessas abelhas.

De modo geral, maior riqueza de Euglossina tem sido encontrada no Panamá (Dodson et al., 1969) e no Brasil na região amazônica (Silveira et al., 2002). No Panamá e Costa Rica foram registradas cerca de $38 \%$ das cerca de 200 espécies descritas, o que equivale à cerca de um terço do total de espécies conhecidas (Roubik e Hanson, 2004).

Os machos de Euglossina visitam flores de algumas famílias botânicas, principalmente Orchidaceae, e nelas coletam substâncias odoríferas (Williams e Whitten, 1983). Segundo Roubik e Hanson (2004) machos e fêmeas visitam flores de pelo menos 23 famílias e nelas coletam néctar. As fêmeas visitam ainda flores de três famílias para extrair resina e nove para coletar pólen. São, por esse motivo, muito importantes para a produção de sementes por essas espécies e manutenção do isolamento reprodutivo e variabilidade genética de suas populaçōes (Mori e Prance, 1987; Bawa, 1990). Contudo, não se sabe a real importância dessas substâncias na biologia dessas abelhas (Williams e Whitten, 1983; Eltz et al., 1999).

O Estado do Acre faz parte do grande arco de desmatamento que ocorre na Amazônia, com $11 \%$ do seu território desmatado (Acre, 2006). Apesar dos poucos estudos de sua biota os resultados indicam haver uma grande diversidade de espécies e endemismos no Estado (Prance, 1973; Souza et al., 2003; Morato e Martins, 2005).

Exceto Morato (2001), Oliveira e Nemésio (2003) e Nemésio e Morato $(2004 ; 2005 ; 2006)$ nenhum outro estudo foi publicado sobre a fauna de Euglossina no Estado do Acre. As amostragens desses trabalhos foram realizadas em poucas áreas e de forma irregular. Contudo, os resultados indicam uma elevada riqueza dessas abelhas na região.

Este trabalho tem como objetivo avaliar a abundância, riqueza, diversidade e composição de espécies de Euglossina na região do Município de Rio Branco, Acre, Amazônia Sul-
Ocidental e comparar esses resultados com os obtidos em outras regióes.

\section{MATERIAL E MÉTODOS}

\section{ÁREAS DE ESTUDO}

O Estado do Acre está localizado no extremo oeste da região norte do Brasil. Possui uma área de 16.422.136,04 ha, cerca de $4 \%$ do território amazônico e $1,9 \%$ do território nacional (Acre, 2006). Na região predomina a floresta tropical de terra firme aberta, bambus, palmeiras e floresta densa (Silveira, 2005). A temperatura média anual varia entre $22^{\circ}$ e $24^{\circ} \mathrm{C}$. O total médio da precipitação anual é $1.973 \mathrm{~mm}$, sendo janeiro o mês mais chuvoso e julho o mais seco (Acre, 2006).

A amostragem foi realizada em 11 áreas florestais, a maioria das quais são áreas de conservação, localizadas nos municípios de Rio Branco, Senador Guiomard, Porto Acre e Bujari: Parque Zoobotânico (221 ha, 9०57’21S; 6752’22W), Círculo Militar (119 ha, 9 ${ }^{\circ} 7^{\prime} 24 S ; 67^{\circ} 48^{\prime} 16 \mathrm{~W}$ ), Horto Florestal (60 ha, 9०56'41S; 67²9’45W), Parque Chico Mendes (65 ha, $10^{\circ} 2^{\prime} 8 S$; $\left.67^{\circ} 47^{\prime} 44 \mathrm{~W}\right)$, Fazenda Experimental Catuaba (1281 ha, $\left.10^{\circ} 4^{\prime} 40 \mathrm{~S} ; 67^{\circ} 37^{\prime} 35 \mathrm{~W}\right)$, Reserva Humaitá (3665 ha, $\left.9^{\circ} 45^{\prime} 17 \mathrm{~S} ; 67^{\circ} 40^{\prime} 15 \mathrm{~W}\right)$, Sítio da Comissão Pró-Índio do Acre (68 ha, $\left.10^{\circ} 00^{\prime} 29 \mathrm{~S} ; 67^{\circ} 54^{\prime} 6 \mathrm{~W}\right)$, Escola da Floresta (698 ha, 959'58S; 6759'14W), Área particular Bujarí (290 ha, $\left.9^{\circ} 49^{\prime} 02 S ; 67^{\circ} 58^{\prime} 18 \mathrm{~W}\right)$, Área de Proteção Ambiental (119 ha, $\left.10^{\circ} 1^{\prime} 29 \mathrm{~S} ; 67^{\circ} 48^{\prime} 33 \mathrm{~W}\right)$ e Secretaria dos Povos Indígenas (0,83 ha, 958'19S; 67\%48'27W).

\section{AMOSTRAGEM}

As amostragens foram realizadas em dois períodos de coleta, o primeiro entre dezembro de 2005 e abril de 2006 e o segundo entre abril de 2006 e setembro de 2006. A duração das coletas em cada área foi de três dias por período, totalizando seis dias por área. Dessa forma, o protocolo amostral foi realizado durante a estação úmida (de maior atividade para a maioria das espécies) e se estendeu até a estação seca, de forma semelhante à amostragem utilizada no Estado do Amazonas por Morato (1994).

As abelhas foram coletadas mediante o emprego de seis substâncias odoríferas: vanilina, cineol, eugenol, metil salicilato, benzil acetato e escatol (Oliveira e Campos, 1996; Nemésio e Morato, 2004).

Em cada área foram escolhidos três pontos de amostragem, distantes cerca de $300 \mathrm{~m}$ um do outro. Nos pontos 1 e 3 foram colocados conjuntos com seis armadilhas confeccionadas com garrafas plásticas de água mineral de $500 \mathrm{ml}$. No ponto 2 foram colocadas seis iscas odoríferas, confeccionadas com cotonetes e a coleta foi realizada com rede entomológica. Nos três pontos as armadilhas ou iscas foram amarradas a um 
fio de náilon, distantes $1 \mathrm{~m}$ uma da outra e a uma altura de aproximadamente $1,5 \mathrm{~m}$ do chão.

As coletas com rede entomológica foram realizadas no período das 07:00 as 13:00 horas. Segundo Oliveira e Campos (1996) este é o horário de maior atividade de Euglossina em florestas tropicais. As armadilhas foram instaladas no primeiro dia de coleta e retiradas no último, permanecendo no local durante os dias de coleta com rede entomológica e sendo recarregadas com as substâncias diariamente.

As abelhas coletadas foram acondicionadas em uma coleção de referência na Universidade Federal do Acre.

\section{ANÁLISE DOS DADOS}

Foram realizadas correlaçōes por intermédio do coeficiente de Pearson (r) e regressóes lineares simples (Sokal e Rohlf, 1995). Para tal, as normalidades das variáveis foram verificadas através do teste de Kolmogorov-Smirnov. Em todas as análises realizadas foi considerado um nível de significância $\alpha=0,05$.

A curva de acumulação de espécies foi calculada conforme a ordem das coletas. Segundo Coleman (1982), qualquer ordem das unidades amostrais é arbitraria. Portanto, para obtenção de uma curva ideal é necessário obter um grande número de curvas e calcular uma curva média. Desta forma, outras duas curvas médias foram calculadas utilizando os estimadores Jackknife e Bootstrap com 50 aleatorizaçôes.

Índices de diversidade de Shannon-Wiener $\left(\mathrm{H}^{\prime}\right)$ e de dominância individual de Berger-Parker (Magurran, 1988) foram calculados para os dados do presente estudo e para os de outras localidades da região Neotropical. Tendo em vista que foram amostradas 11 áreas, foram consideradas mais comuns as espécies para as quais foram coletados pelo menos 11 indivíduos, de tal forma que para estas espécies, por chance, pelo menos um indivíduo poderia ser amostrado em cada área.

\section{RESULTADOS}

Foram coletados 3.675 machos de Euglossina pertencentes a quatro gêneros e 36 espécies (Tabela 1). Destes, 1.789 $(48,7 \%)$ pertencentes a 34 espécies foram capturados com rede entomológica e $1.886(51,3 \%)$ pertencentes a 24 espécies foram coletados com armadilhas. Eulaema cingulata (Fabricius), 1804 (24,6\%) foi a espécie mais comum, seguida por Eulaema meriana (Olivier), 1789 (14,6\%), Euglossa amazonica Dressler, 1982 (10,5\%), Eulaema nigrita Lepeletier, 1841 (9,7\%) e Eulaema pseudocingulata (Oliveira), 2006 (7,2\%). Dentre os indivíduos coletados com rede entomológica 55,7\% pertencem ao gênero Euglossa, 39,2\% ao gênero Eulaema, 6,5\% ao gênero Eufriesea e 4,2\% ao gênero Exaerete. Nas coletas com armadilhas $78,5 \%$ dos indivíduos capturados pertencem ao gênero Eulaema, 14,8\% ao gênero
Euglossa, 5,5\% ao gênero Exaerete e 1,2\% ao gênero Eufriesea. Das 36 espécies oito foram coletadas exclusivamente com rede entomológica e duas exclusivamente nas armadilhas.

O número acumulado de espécies coletadas estabilizou a partir da $48^{\mathrm{a}}$ coleta (Figura 1). Poucas espécies foram abundantes, sendo a maioria representada por menos de 50 indivíduos (Figura 2).

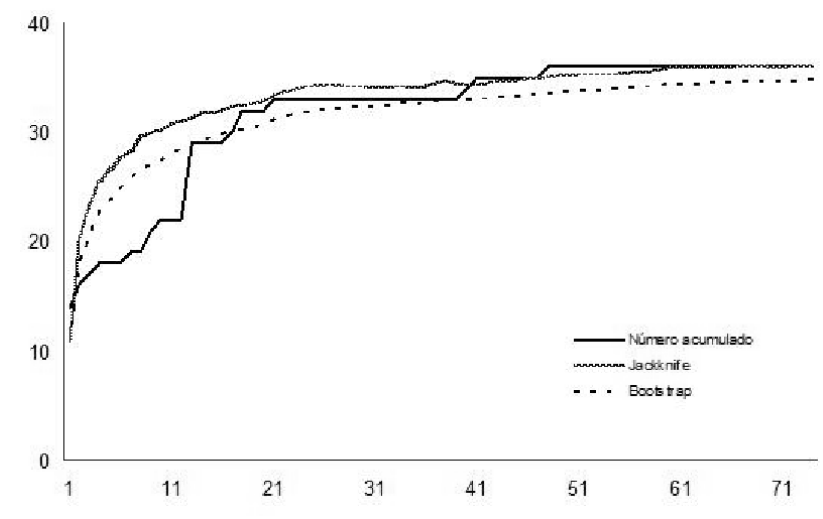

Figura 1 - Número acumulado de espécies de abelhas Euglossina durante 0 período amostral em áreas florestais na região de Rio Branco, Acre, Amazônia Sul-Ocidental.

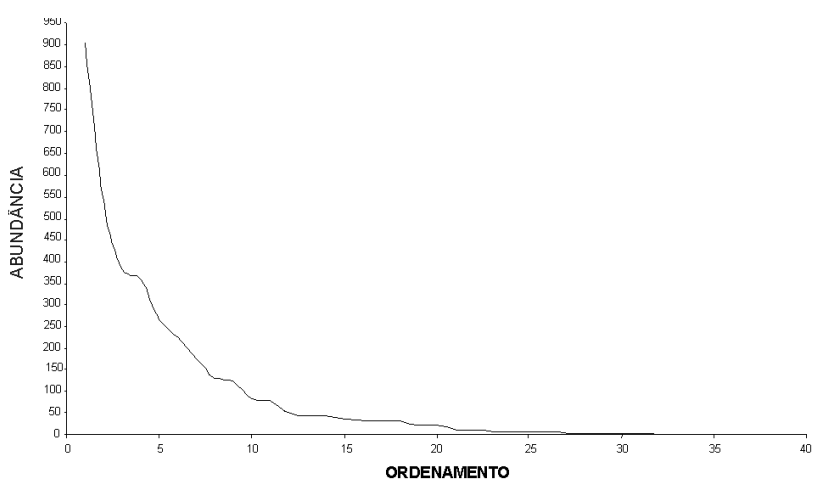

Figura 2 - Ordenamento em abundância das espécies de Euglossina coletadas em áreas florestais na região de Rio Branco, Acre, Amazônia Sul-Ocidental.

Nas coletas com rede entomológica, maior número de indivíduos foram atraídos entre as 9:30 e 10:30 hs, com pico entre 10:00 e 10:30 hs (Figura 3).

Das seis substâncias odoríferas utilizadas, cineol atraiu maior número de indivíduos $(23,8 \%)$ seguida por vanilina $(19,9 \%)$ e benzil acetato $(19,4 \%)$ (Tabela 2). Porém, metil salicilato $(77,7 \%)$ e cineol $(66,6 \%)$ atraíram maior número de espécies. Do total de indivíduos coletados 476 (12,9\%) não definiram uma isca odorífera, sendo capturados quando em vôo próximo às iscas ou sobre a vegetação. Não houve correlação significativa $(r=-0,320 ; p=0,946 ;$ g.l. $=4)$ entre a abundância e riqueza por isca odorífera. 
Tabela 1 - Abundância e riqueza de Euglossina coletadas em áreas florestais na Amazônia Sul-0cidental.

\begin{tabular}{|c|c|c|c|}
\hline ESPÉCIE & REDE & ARMADILHA & TOTAL \\
\hline Eufriesea flaviventris (Friese), 1899 & 5 & 3 & 8 \\
\hline Ef. ornata (Mocsáry), 1896 & 2 & & 2 \\
\hline Ef. pulchra (Smith), 1854 & 4 & 5 & 9 \\
\hline Ef. superba (Hoffmannsegg), 1817 & & 8 & 8 \\
\hline Ef. surinamensis (Linnaeus), 1758 & 5 & 6 & 11 \\
\hline Euglossa allosticta Moure, 1969 & 38 & 13 & 51 \\
\hline Eg. amazonica Dressler, 1982 & 283 & 103 & 386 \\
\hline Eg. analis Westwood, 1840 & 1 & & 1 \\
\hline Eg. augaspis Dressler, 1982 & 31 & 13 & 44 \\
\hline Eg. avicula Dressler, 1982 & 2 & & 2 \\
\hline Eg. bidentata Dressler, 1982 & 21 & 14 & 35 \\
\hline Eg. chlorina Dressler, 1982 & 21 & 2 & 23 \\
\hline Eg. cognata Moure, 1970 & 29 & 3 & 32 \\
\hline Eg. crassipunctata Moure, 1968 & & 1 & 1 \\
\hline Eg. gaianii Dressler, 1982 & 1 & & 1 \\
\hline Eg. ignita Smith, 1874 & 144 & 30 & 174 \\
\hline Eg. imperialis Cockerell, 1922 & 40 & 4 & 44 \\
\hline Eg. intersecta Latreille, 1938 & 3 & & 3 \\
\hline Eg. iopyrrha Dressler, 1982 & 1 & & 1 \\
\hline Eg. magnipes Dressler, 1982 & 4 & & 4 \\
\hline Eg. mixta Friese, 1899 & 64 & 23 & 87 \\
\hline Eg. modestior Dressler, 1982 & 183 & 40 & 223 \\
\hline Eg. mourei Dressler, 1982 & 105 & 29 & 134 \\
\hline Eg. orellana Roubik, 2004 & 11 & 2 & 13 \\
\hline Eg. prasina Dressler, 1982 & 11 & 2 & 13 \\
\hline Eg. securigera Dressler, 1982 & 3 & 1 & 4 \\
\hline Eulaema bombiformis (Packard), 1869 & 2 & 36 & 38 \\
\hline El. cingulata (Fabricius), 1804 & 296 & 607 & 903 \\
\hline El. meriana (Olivier), 1789 & 114 & 424 & 538 \\
\hline El. mocsaryi (Friese), 1899 & 50 & 30 & 80 \\
\hline El. nigrita Lepeletier, 1841 & 124 & 233 & 357 \\
\hline El. pseudocingulata (Oliveira), 2006 & 115 & 150 & 265 \\
\hline Exaerete dentata (Linnaeus), 1758 & 1 & & 1 \\
\hline Ex. frontalis (Guérin-Méneville), 1845 & 11 & 11 & 22 \\
\hline Ex. lepeletieri Oliveira e Nemésio, 2003 & 10 & 24 & 34 \\
\hline Ex. smaragdina (Guérin-Méneville), 1845 & 54 & 69 & 123 \\
\hline Abundância & 1.789 & 1.886 & 3.675 \\
\hline Riqueza & 34 & 28 & 36 \\
\hline Espécies exclusivas por método & 8 & 2 & - \\
\hline
\end{tabular}


Tabela 2 - Abundância e riqueza de Euglossina coletadas em diferentes iscas odoríferas na Amazônia Sul-Ocidental. $\mathrm{BA}=$ benzil acetato; $\mathrm{Cl}=$ cineol; $\mathrm{ES}=$ escatol; $\mathrm{EU}=$ eugenol; $\mathrm{MS}$ = metil salicilato; $\mathrm{VA}$ = vanilina; $\mathrm{ND}$ = foram coletados no ponto de coleta, porém não nas iscas.

\begin{tabular}{|c|c|c|c|c|c|c|c|c|}
\hline ESPÉCIE & $\mathrm{BA}$ & $\mathrm{Cl}$ & ES & EU & MS & VA & ND & TOTAL \\
\hline Eufriesea flaviventris & 1 & & & & 6 & 1 & & 8 \\
\hline Ef. ornata & & & & & 1 & & 1 & 2 \\
\hline Ef. pulchra & & & & 1 & 6 & & 2 & 9 \\
\hline Ef. surinamensis & & & 3 & & 1 & 5 & 2 & 11 \\
\hline Ef. superba & 1 & 1 & & & & 6 & & 8 \\
\hline Euglossa allosticta & & 22 & 19 & 2 & & 1 & 7 & 51 \\
\hline Eg. amazonica & 11 & 267 & 62 & 9 & 3 & 3 & 31 & 386 \\
\hline Eg. analis & & & 1 & & & & & 1 \\
\hline Eg. augaspis & & 8 & 5 & 7 & 2 & 19 & 3 & 44 \\
\hline Eg. avicula & & 1 & & & & 1 & & 2 \\
\hline Eg. bidentata & 3 & 2 & 8 & 1 & 3 & 13 & 5 & 35 \\
\hline Eg. chlorina & 1 & 11 & 3 & 2 & 1 & & 5 & 23 \\
\hline Eg. cognata & 5 & 1 & & 1 & 17 & & 8 & 32 \\
\hline Eg. crassipunctata & & & & & & 1 & & 1 \\
\hline Eg. gaianii & & & & & 1 & & & 1 \\
\hline Eg. ignita & 23 & 71 & 11 & 6 & 26 & & 37 & 174 \\
\hline Eg. imperialis & 1 & 21 & 1 & & 11 & 2 & 8 & 44 \\
\hline Eg. intersecta & & 1 & & & & & 2 & 3 \\
\hline Eg. iopyrrha & & & & & 1 & & & 1 \\
\hline Eg. magnipes & & & & 1 & & & 3 & 4 \\
\hline Eg. mixta & 2 & 1 & 13 & 3 & 42 & 2 & 23 & 86 \\
\hline Eg. modestior & 4 & 194 & 1 & 4 & 2 & 2 & 16 & 223 \\
\hline Eg. mourei & 4 & 61 & 4 & & 1 & 53 & 11 & 134 \\
\hline Eg. orellana & & 8 & 1 & 1 & 1 & & 2 & 13 \\
\hline Eg. prasina & & & 1 & & 1 & 6 & 5 & 13 \\
\hline Eg. securigera & & 2 & & & 1 & & 1 & 4 \\
\hline Eulaema bombiformis & 10 & & & & 24 & 2 & 2 & 38 \\
\hline El. cingulata & 384 & 9 & 7 & 33 & 2 & 314 & 154 & 903 \\
\hline El. meriana & 215 & 30 & 8 & 2 & 212 & 47 & 24 & 538 \\
\hline El. mocsaryi & 24 & 1 & 3 & 5 & 12 & 16 & 19 & 80 \\
\hline El. nigrita & 3 & 43 & 157 & 3 & 6 & 111 & 34 & 357 \\
\hline El. pseudocingulata & 17 & 7 & 29 & 47 & 2 & 121 & 42 & 265 \\
\hline Exaerete dentata & & & & & & & 1 & 1 \\
\hline Ex. frontalis & 1 & 15 & 1 & 2 & 2 & & 2 & 23 \\
\hline Ex. lepeletieri & & 21 & 1 & 1 & 7 & 1 & 3 & 34 \\
\hline Ex. smaragdina & 3 & 78 & 1 & 8 & 4 & 6 & 23 & 123 \\
\hline Abundância & 713 & 876 & 340 & 139 & 398 & 733 & 476 & 3.675 \\
\hline Riqueza & 19 & 24 & 22 & 20 & 28 & 22 & 29 & 36 \\
\hline
\end{tabular}




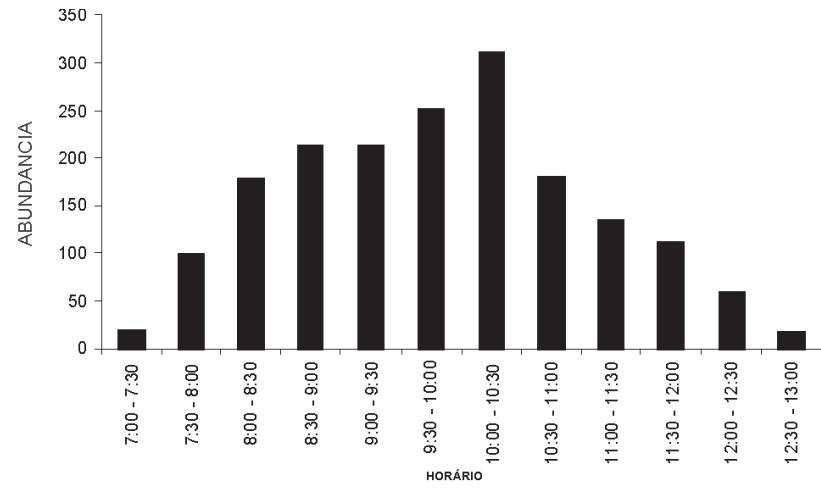

Figura 3 - Horário de atividade de machos de Euglossina atraídos por iscas odoríferas e coletados com rede entomológica durante o período amostral em áreas florestais na região de Rio Branco, Acre, Amazônia Sul-Ocidental.

Para ambos os métodos cineol e metil salicilato atraíram maior abundância e riqueza, respectivamente, tanto nas coletas com rede entomológica quanto nas com armadilhas. Não houve correlação significativa entre abundância de abelhas coletadas com armadilhas e com rede entomológica por substância odorífera $(r=0,1845 ; \mathrm{p}=0,0726 ; \mathrm{g} .1 . \mathrm{l}=4)$. O mesmo ocorreu em relação à riqueza $(r=0,6469 ; p=0,165$; g.l. = 4).

Foram coletados $31(0,8 \%)$ indivíduos pertencentes a nove espécies portando polinários de orquídeas (Tabela 3). Destes, $27(87,1 \%)$ pertencem a seis espécies de Eulaema e quatro $(12,9 \%)$ a três espécies de Euglossa. A substância que mais atraiu indivíduos com polinários foi eugenol $(22,5 \%)$ e cerca de $61 \%$ dos indivíduos foram coletados nas armadilhas.

A abundância, riqueza, diversidade, dominância e métodos de coleta de trabalhos realizados em diversas localidades da região Neotropical são mostrados na Tabela 4. Porém, alguns dados não foram aqui contemplados por não terem sido apresentados pelos autores em seus trabalhos. A abundância de abelhas coletada pelos diferentes autores apresentou correlação positiva e significativa com a riqueza de espécies $(\mathrm{r}=0,51 ; \mathrm{p}$ $=0,004 ;$ g.l. $=28)($ Figura 4A). Houve correlação significativa e negativa entre a dominância e diversidade $(r=0,89 ; \mathrm{p}=$ 0,000; g.l. = 28) (Figura 4B).

\section{DISCUSSÃO}

O conhecimento sobre a riqueza de espécies de Euglossina em algumas regiōes do Brasil como Centro-Oeste, Nordeste e Amazônica ainda é muito fragmentado. Na região amazônica, pelo menos 47 espécies foram listadas (Morato et al., 1992; 1994; Oliveira e Campos, 1995; Nemésio, 2005).

Para o Estado do Acre, Morato (2001) e Nemésio e Morato (2004; 2005; 2006) registraram 46 espécies baseados em coletas nos município de Rio Branco e arredores e no Parque Nacional da Serra do Divisor, localizado a noroeste do Estado.

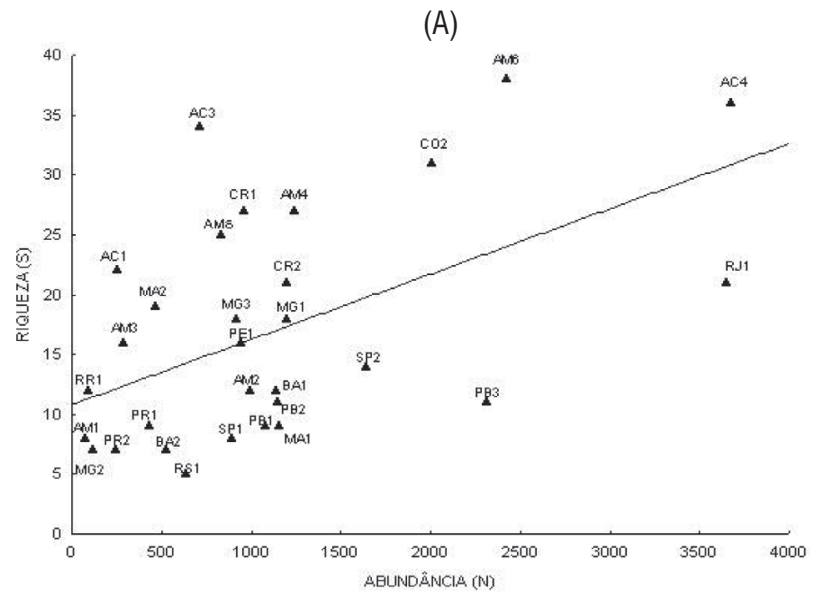

(B)

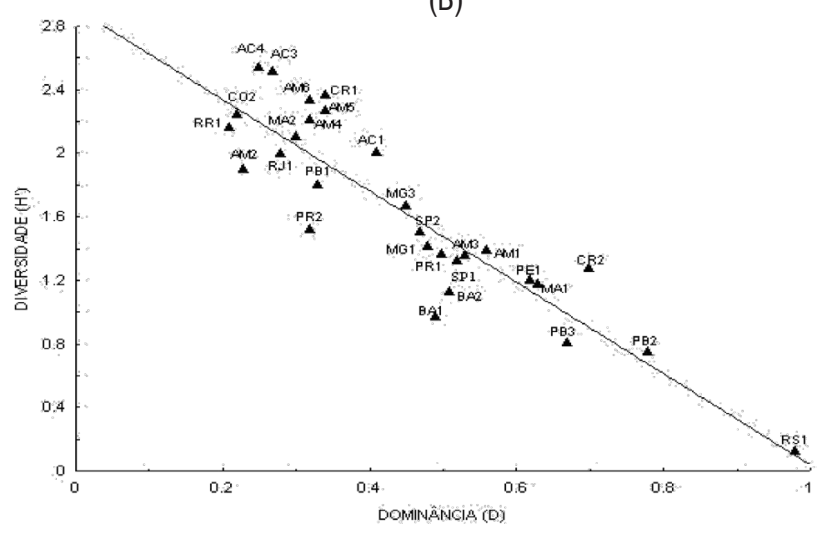

Figura 4 - Relação entre riqueza e abundância (A) e diversidade e dominância (B) de abelhas Euglossina em diferentes localidades da região Neotropical. As linhas retas ajustadas têm apenas propósito ilustrativo.AM1 = Braga (1976); CR1 = Janzen et al. (1982); CR2 = Janzen et al. (1982); AM2 = Powell \& Powell (1987); RS1 = Wittmann et al. (1988); SP1 = Rebêlo \& Garófalo (1991); AM3 = Becker et al. (1991); AM4 = Morato et al. (1992); AM5 = Morato (1994); AM6 = Oliveira \& Campos (1995); SP2 = Rebêlo e Garófalo (1997); BA1 = Neves \& Viana (1997); MA1 = Rebêlo \& Cabral (1997); BA2 $=$ Neves \& Viana (1999); MG1 = Peruquetti et al. (1999); PB1 = Bezerra \& Martins (2001); MA2 = Brito \& Rego (2001); RJ1 = Tonhasca et al. (2002); MG2 = Nemésio (2003); C02 = Otero \& Sandino (2003); AC1 = Nemésio \& Morato (2004); PR1 = Sofia et al.(2004); PR2 = Sofia \& Suzuki (2004); PB2 $=$ Martins \& Souza (2005); PE1 = Milet-Pinheiro \& Schlindwein (2005); RR1 $=$ Nemésio (2005); PB3 = Souza et al. (2005); MG3 = Nemésio \& Silveira (2006); AC3 = Nemésio \& Morato (2006); AC4 = este estudo.

O presente trabalho registrou a presença de mais oito espécies no Estado (Eufriesea surinamensis (Linnaeus), 1758, Euglossa avicula Dressler, 1982, Euglossa gaianii Dressler, 1982, Euglossa iopyrrha Dressler, 1982 Euglossa magnipes Dressler, 1982 Euglossa prasina Dressler, 1982 Euglossa securigera Dressler, 1982 e Exaerete dentata (Linnaeus), 1758 aumentando a lista de Euglossina do Acre para 52 espécies.

El. cingulata e El. meriana que foram as espécies mais comuns neste trabalho foram também as mais coletadas por 
Tabela 3 - Machos de Euglossina coletados portando polinários de orquídeas em áreas florestais na Amazônia Sul-0cidental. $A=$ armadilha; $R=$ rede entomológica; $\mathrm{BA}$ = benzil acetato; $\mathrm{Cl}$ = cineol; $\mathrm{ES}$ = escatol; $\mathrm{EU}=$ eugenol; $\mathrm{MS}$ = metil salicilato; $\mathrm{VA}$ = vanilina; $\mathrm{ND}=$ foram coletados no ponto de coleta, porém não nas iscas.

\begin{tabular}{|c|c|c|c|c|c|}
\hline ESPÉCIE & $\begin{array}{l}\text { DATA DE } \\
\text { COLETA }\end{array}$ & MÉTODO & HORÁRIO & SUBSTÂNCIA & No. POLINÁRIOS \\
\hline Eulaema nigrita Lepeletier & $09 / 03 / 06$ & A & - & VA & 1 \\
\hline El. nigrita & $09 / 05 / 06$ & A & - & VA & 1 \\
\hline El. nigrita & $18 / 05 / 06$ & A & - & ES & 1 \\
\hline El. nigrita & $19 / 05 / 06$ & A & - & VA & 1 \\
\hline El. nigrita & $10 / 06 / 06$ & A & - & $\mathrm{Cl}$ & 1 \\
\hline El. nigrita & $10 / 06 / 06$ & A & - & VA & 1 \\
\hline El. nigrita & $20 / 06 / 06$ & A & - & ES & 1 \\
\hline El. cingulata (Fabricius) & $20 / 04 / 06$ & $\mathrm{R}$ & $11: 30-12: 00$ & VA & 1 \\
\hline El. cingulata & $20 / 04 / 06$ & $\mathrm{R}$ & $10: 30-11: 00$ & $\mathrm{Cl}$ & 1 \\
\hline El. cingulata & $19 / 06 / 06$ & $\mathrm{R}$ & 07:00 - 07:30 & EU & 1 \\
\hline El. cingulata & $25 / 06 / 06$ & A & - & EU & 1 \\
\hline El. pseudocingulata (Oliveira) & $19 / 12 / 06$ & $\mathrm{R}$ & $10: 30-11: 00$ & EU & 1 \\
\hline El. pseudocingulata & $05 / 04 / 06$ & A & - & EU & 1 \\
\hline El. pseudocingulata & 05/04/06 & A & - & EU & 1 \\
\hline El. pseudocingulata & $04 / 05 / 06$ & $\mathrm{R}$ & 09:30 - 10:00 & EU & 1 \\
\hline El. pseudocingulata & $09 / 05 / 06$ & $\mathrm{R}$ & $10: 30-11: 00$ & EU & 1 \\
\hline El. pseudocingulata & $19 / 06 / 06$ & A & - & BA & 1 \\
\hline El. pseudocingulata & $19 / 06 / 06$ & $\mathrm{R}$ & 07:30 - 08:00 & ND & 1 \\
\hline El. meriana (Olivier) & $20 / 04 / 06$ & $\mathrm{R}$ & 09:30 - 10:00 & BA & 1 \\
\hline El. meriana & $20 / 04 / 06$ & $\mathrm{R}$ & 09:30 - 10:00 & ND & 1 \\
\hline El. meriana & 09/05/06 & A & - & MS & 1 \\
\hline El. meriana & $24 / 05 / 06$ & A & - & MS & 2 \\
\hline El. meriana & $05 / 06 / 06$ & A & - & MS & 1 \\
\hline El. meriana & $07 / 05 / 06$ & A & - & BA & 1 \\
\hline El. bombiformis (Packard) & $01 / 07 / 06$ & A & - & MS & 2 \\
\hline El. mocsaryi (Friese) & $26 / 04 / 06$ & $\mathrm{R}$ & 08:00 -08:30 & BA & 1 \\
\hline El. mocsaryi & $07 / 06 / 06$ & A & - & BA & 1 \\
\hline Euglossa ignita Smith & $26 / 05 / 06$ & $\mathrm{R}$ & 08:30 - 09:00 & $\mathrm{Cl}$ & 1 \\
\hline Eg. ignita & $04 / 07 / 06$ & A & - & $\mathrm{Cl}$ & 1 \\
\hline Eg. amazonica Dressler & $21 / 04 / 06$ & A & $11: 00-11: 30$ & ES & 1 \\
\hline Eg. modestior Dressler & $15 / 06 / 06$ & $\mathrm{R}$ & 09:00 - 09:30 & $\mathrm{Cl}$ & 1 \\
\hline Total de indivíduos & & & & & 31 \\
\hline Total de espécies & & & & & 9 \\
\hline
\end{tabular}

Nemésio e Morato (2004; 2005; 2006) no Estado do Acre. Nemésio (2003) e Nemésio e Silveira (2006) encontraram maior abundância de El. cingulata em Minas Gerais e El. meriana foi muito coletada por Morato (1994) em áreas de mata e capoeiras no Amazonas. Eg. amazonica não foi abundante em outras coletas realizadas no Acre e Becker $e$ t al. (1991) registraram apenas um indivíduo na Amazônia Central. El. nigrita, considerada bioindicadora de áreas impactadas (Morato, 1994), foi muito coletada nas regiōes sul, sudeste e nordeste (Neves e Viana, 1997; Rebêlo e Garófalo, 1997; Peruquetti et al., 1999; Bezerra e Martins, 2001; Nemésio, 2003; Sofia e Suzuki, 2004; Souza et al., 2005). Esta espécie foi rara nos trabalhos de Nemésio e Morato (2004) e Nemésio (2005) e não foi registrada nos trabalhos feitos em fragmentos florestais no Amazonas (Powell e Powell 1987; Becker et al., 1991; Oliveira e Campos, 1996), talvez por esses fragmentos estarem envolvidos por capoeiras e pastagens. Entretanto, esta espécie foi uma das mais abundantes neste trabalho. Isso 
Tabela 4 - Abundância, riqueza e diversidade de Euglossina em diferentes localidades da região Neotropical.

\begin{tabular}{|c|c|c|c|c|c|c|c|}
\hline REFERÊNCIA & LOCAL & $\begin{array}{c}\text { ABUNDÂNCIA } \\
(\mathrm{N})\end{array}$ & $\begin{array}{l}\text { RIQUEZA } \\
\text { (S) }\end{array}$ & $\begin{array}{l}\text { DIVERSIDADE } \\
\left(\mathrm{H}^{\prime}\right)\end{array}$ & $\begin{array}{l}\text { DOMINÂNCIA } \\
\text { (D) }\end{array}$ & ESPÉCIE DOMINANTE & $\begin{array}{c}\text { MÉTODO } \\
\text { DE } \\
\text { COLETA }\end{array}$ \\
\hline Dodson et al. (1969) & Panamá & 927 & 48 & $\S$ & $\S$ & $\dagger$ & $\mathrm{R}$ \\
\hline Dodson et al. (1969) & Colombia Leste & 160 & 42 & $\S$ & $\S$ & $\dagger$ & $\mathrm{R}$ \\
\hline Braga (1976) & Brasil, AM & 76 & 8 & 1,39 & 0,56 & Euglossa ignita Smith, & $\mathrm{R}$ \\
\hline Janzen et al. (1982) & Costa Rica,Corcovado & 961 & 27 & 2,37 & 0,34 & Eg. imperialis Cockerell & $\mathrm{R}$ \\
\hline Janzen et al. (1982) & Costa Rica, Santa Rosa & 1200 & 21 & 1,28 & 0,70 & $\begin{array}{c}\text { Euglossa viridissima } \\
\text { Friese }\end{array}$ & $\mathrm{R}$ \\
\hline Ackerman (1983) & Panamá & 21.842 & 44 & 2,43 & 0,26 & $\begin{array}{c}\text { Euglossa tridentata } \\
\text { Moure }\end{array}$ & $\mathrm{R}$ \\
\hline Pearson e Dressler (1985) & Peru & 2.917 & 38 & $\S$ & $\S$ & $\dagger$ & $\mathrm{R}$ \\
\hline Powell e Powell (1987) & Brasil, AM & 992 & 12 & 1,90 & 0,23 & $\begin{array}{c}\text { Euglossa chalybeata } \\
\text { Friese }\end{array}$ & 0 \\
\hline Roubik e Ackerman (1987) & Panamá & 20.000 & 51 & $\S$ & $\S$ & $\dagger$ & A \\
\hline Wittmann et al. (1988) & Brasil, RS & 639 & 5 & 0,13 & 0,98 & $\begin{array}{c}\text { Eufriesea violacea } \\
\text { (Blanchard) }\end{array}$ & $\mathrm{R}$ \\
\hline Ackerman (1989) & Panamá & 27.874 & 53 & $\S$ & $\S$ & $\dagger$ & A \\
\hline Rebêlo e Garófalo (1991) & Brasil, SP & 892 & 8 & 1,33 & 0,52 & $\begin{array}{c}\text { Euglossa pleosticta } \\
\text { Dressler }\end{array}$ & $\mathrm{R}$ \\
\hline Becker et al. (1991) & Brasil, AM & 290 & 16 & 1,36 & 0,53 & $\begin{array}{c}\text { Euglossa stilbonota } \\
\text { Dressler }\end{array}$ & A \\
\hline Morato et al. (1992) & Brasil, AM & 1.242 & 27 & 2,21 & 0,32 & $\begin{array}{c}\text { Euglossa stilbonota } \\
\text { Dressler }\end{array}$ & A \\
\hline Morato (1994) & Brasil, AM & 838 & 25 & 2,27 & 0,34 & $\begin{array}{c}\text { Euglossa stilbonota } \\
\text { Dressler }\end{array}$ & A \\
\hline Oliveira e Campos (1995) & Brasil, AM & 2.422 & 38 & 2,34 & 0,32 & $\begin{array}{c}\text { Euglossa stilbonota } \\
\text { Dressler }\end{array}$ & A \\
\hline Rebêlo e Garófalo (1997) & Brasil, SP & 1.642 & 14 & 1,51 & 0,47 & $\begin{array}{c}\text { Euglossa pleosticta } \\
\text { Dressler }\end{array}$ & $\mathrm{R}$ \\
\hline Neves e Viana (1997) & Brasil, BA & 1.144 & 12 & 0,97 & 0,49 & Eulaema nigrita Lepeletier & A \\
\hline Rebêlo e Cabral (1997) & Brasil, MA & 1.153 & 9 & 1,18 & 0,63 & $\begin{array}{l}\text { Euglossa cordata } \\
\text { (Linnaeus) }\end{array}$ & $\mathrm{R}$ \\
\hline Neves e Viana (1999) & Brasil, BA & 527 & 7 & 1,13 & 0,51 & $\begin{array}{l}\text { Euglossa cordata } \\
\text { (Linnaeus) }\end{array}$ & $A / R$ \\
\hline Peruquetti et al. (1999) & Brasil, MG & 1.201 & 18 & 1,42 & 0,48 & Eulaema nigrita Lepeletier & $\mathrm{A} / \mathrm{N}$ \\
\hline Bezerra e Martins (2001) & Brasil, PB & 1.082 & 9 & 1,80 & 0,33 & Eulaema nigrita Lepeletier & A \\
\hline Brito e Rêgo (2001) & Brasil, MA & 467 & 19 & 2,11 & 0,30 & $\begin{array}{c}\text { Euglossa piliventris } \\
\text { Guérin }\end{array}$ & $\mathrm{R}$ \\
\hline Tonhasca et al. (2002) & Brasil, RJ & 3.653 & 21 & 2,00 & 0,28 & Eulaema nigrita Lepeletier & $\mathrm{R} / \mathrm{N}$ \\
\hline Nemésio (2003) & Brasil, MG & 122 & 7 & 1,48 & 0,41 & $\begin{array}{l}\text { Eulaema cingulata } \\
\text { (Fabricius) }\end{array}$ & $\mathrm{R}$ \\
\hline Otero e Sandino (2003) & Colombia & 2.008 & 31 & 2,25 & 0,22 & Euglossa ignita Smith & A \\
\hline Nemésio e Morato (2004) & Brasil, AC & 254 & 22 & 2,01 & 0,41 & $\begin{array}{l}\text { Eulaema cingulata } \\
\text { (Fabricius) }\end{array}$ & $A / R$ \\
\hline Sofia et al. (2004) & Brasil, PR & 434 & 9 & 1,37 & 0,50 & $\begin{array}{l}\text { Eufriesea violacea } \\
\quad \text { (Blanchard) }\end{array}$ & $\mathrm{R}$ \\
\hline Sofia e Suzuki (2004) & Brasil, PR & 245 & 7 & 1,52 & 0,32 & $\begin{array}{l}\text { Eufriesea violacea } \\
\text { (Blanchard) }\end{array}$ & $\mathrm{R}$ \\
\hline Martins e Souza (2005) & Brasil, PB & 1.151 & 11 & 0,75 & 0,78 & Eulaema nigrita Lepeletier & A \\
\hline $\begin{array}{l}\text { Milet-Pinheiro e Schlindwein } \\
(2005)\end{array}$ & Brasil, PE & 945 & 16 & 1,20 & 0,62 & $\begin{array}{l}\text { Euglossa cordata } \\
\text { (Linnaeus) }\end{array}$ & $\mathrm{R}$ \\
\hline Nemésio (2005) & Brasil, RR & 90 & 12 & 2,16 & 0,21 & $\begin{array}{l}\text { Eufriesea superba } \\
\text { (Hoffmannsegg) }\end{array}$ & A \\
\hline
\end{tabular}




\begin{tabular}{|c|c|c|c|c|c|c|c|}
\hline REFERÊNCIA & LOCAL & $\begin{array}{c}\text { ABUNDÂNCIA } \\
(\mathrm{N})\end{array}$ & $\begin{array}{l}\text { RIQUEZA } \\
\text { (S) }\end{array}$ & $\begin{array}{c}\text { DIVERSIDADE } \\
\left(\mathrm{H}^{\prime}\right)\end{array}$ & $\begin{array}{l}\text { DOMINÂNCIA } \\
\text { (D) }\end{array}$ & ESPÉCIE DOMINANTE & $\begin{array}{c}\text { MÉTODO } \\
\text { DE } \\
\text { COLETA }\end{array}$ \\
\hline Souza et al. (2005) & Brasil, PB & 2.314 & 11 & 0,81 & 0,67 & $\begin{array}{l}\text { Euglossa cordata } \\
\text { (Linnaeus) }\end{array}$ & A \\
\hline Nemésio e Silveira (2006) & Brasil, MG & 918 & 18 & 1,67 & 0,45 & $\begin{array}{c}\text { Euglossa analis } \\
\text { Westwood }\end{array}$ & $\mathrm{R}$ \\
\hline Nemésio e Morato (2005) & Brasil, AC & 541 & 30 & $\dagger$ & $\dagger$ & $\dagger$ & \\
\hline Nemésio e Morato (2206) & Brasil, AC & 715 & 34 & 2,52 & 0,27 & $\begin{array}{l}\text { Eulaema cingulata } \\
\text { (Fabricius) }\end{array}$ & $\mathrm{R} / \mathrm{A}$ \\
\hline Este estudo & Brasil, AC & 3.675 & 36 & 2,54 & 0,25 & $\begin{array}{l}\text { Eulaema cingulata } \\
\text { (Fabricius) }\end{array}$ & $\mathrm{R} / \mathrm{A}$ \\
\hline
\end{tabular}

$A=$ armadilha; $R=$ rede; 0 = Não envolveu coleta, apenas observação; $N=$ ninho-armadilha; $\S=$ não contém dados que permitam 0 cálculo dos índices; $†=$ não informado.

talvez se deva ao fato de que metade das áreas aqui amostradas estão inseridas na sede do Município de Rio Branco. Somente seis indivíduos de El. pseudocingulata, espécie que no presente trabalho foi a quinta mais abundante, foram registrados por Nemésio e Morato (2004). Porém, essa espécie não foi registrada nos demais trabalhos realizados na região por ter sido descrita recentemente (Oliveira, 2006).

Nemésio e Morato (2004) verificaram que as coletas com rede entomológica podem ser cerca de quatro vezes mais eficiente tanto para abundância quanto para riqueza, do que as coletas com armadilhas. Contudo, neste trabalho, maior número de indivíduos foi coletado nas armadilhas. Possivelmente, isto se deve ao fato de que estas permaneceram no local durante todos os dias de coleta enquanto as iscas permaneceram das 07:00 as 13:00 horas. As armadilhas coletaram grande quantidade de indivíduos pertencentes ao gênero Eulaema enquanto nas coletas com rede entomológica a maioria foi do gênero Euglossa. Isso pode estar associado ao tamanho corporal, pois, as Euglossa são de menor tamanho e conseguem escapar mais facilmente pelas aberturas das armadilhas, o mesmo não ocorrendo para as Eulaema que são de tamanhos maiores. Outra possibilidade é que machos de espécies consideradas mais agressivas impedem que outras espécies entrem nas armadilhas para coletar substâncias (Nemésio e Morato 2004).

Algumas espécies como Eufriesea ornata (Mocsáryi), 1896 Euglossa analis Westwood, 1840 Eg. iopyrrha, Eg. avicula, Eg. gaianii, Euglossa intersecta Latreille, 1938, Eg. magnipes e Ex. dentata foram coletadas neste trabalho somente com rede entomológica. Eufriesea superba (Hoffmannsegg), 1817 e Euglossa crassipunctata Moure, 1968 foram coletadas somente nas armadilhas. Um indivíduo de Aglae caerulea Lepeletier e Serville, 1825 espécie muito rara de se encontrar, foi coletado em armadilha por Morato (2001) no Parque Nacional da Serra do Divisor. Dessa forma a utilização dos dois métodos de coleta pode ser importante em inventários faunísticos dessas abelhas.
As armadilhas podem ser utilizadas como complementos das coletas com rede entomológica.

Um macho de Ex. dentata, espécie até então registrada apenas na região sudeste (Silveira et al., 2002) foi também coletado neste estudo.

O número acumulado de espécies estabilizou próximo da $48^{a}$ coleta sendo que as espécies consideradas comuns foram coletadas logo nas primeiras amostras. Portanto, o esforço amostral empreendido foi suficiente para amostrar a diversidade regional dessas abelhas. Algumas espécies de Eufriesea são altamente sazonais, sendo ativas durante 1 ou 2 meses no ano (Dressler, 1982). Consequentemente, protocolos amostrais mais longos são mais eficientes para o conhecimento da fauna local. A separação temporal e espacial das coletas durante o período amostral também deve ter contribuído para a captura de maior riqueza de espécies de Euglossina, haja visto que foram coletados 38 indivíduos pertencentes a 5 espécies de Eufriesea.

Os estudos realizados nas regióes sul e sudeste mostram baixa diversidade de espécies de Euglossina e elevada dominância. O mesmo não ocorreu neste estudo onde a diversidade foi elevada e foram poucas as espécies dominantes, o que parece ser um padrão para região amazônica (Roubik e Hanson, 2004). Tonhasca et al. (2002) encontrou resultado semelhante, porém em florestas de Mata Atlântica.

A maioria dos indivíduos foi coletada entre 8:30 e 10:30 horas. Oliveira (1999) verificou maior atratividade das iscas a partir das 9:00 horas. Em um segundo ponto de coleta verificou um pico na abundância e riqueza de indivíduos às 11:00 horas. Segundo esse autor a temperatura local e a oferta de substâncias em fontes naturais pode influenciar na atividade dos machos fazendo com que estes sejam mais ativos em determinados horários. Bezerra e Martins (2001) na região Nordeste, verificaram maior atividade entre 8:00 e 9:00 horas. Na região Sul Wittmann et al. (1989) verificaram que a atividade diária aumentou gradualmente das 9:00 as 11:00 horas. 
Contudo, Soares et al. (1989) verificaram que machos de Euglossa mandibularis Friese, 1899 coletam substâncias em flores de Cyphomandra calycina Sendt (Solanaceae) às 5:30 horas. Braga e Garófalo (2003) verificaram a presença de machos de Euglossa towsendii Cockerell, 1904 em flores de Crinum procerum Carey (Amaryllidaceae) das 7:30 às 16:00 horas.

Cineol foi a isca que atraiu maior número de indivíduos e metil salicilato o maior número de espécies. Resultados semelhantes foram encontrados por Oliveira e Campos (1996), Powell e Powell (1987) e em Morato (1992) nos quais cineol atraiu maior número de indivíduos e foi atrativo para todas as espécies coletadas juntamente com metil salicilato. Cineol atraiu maior abundância e riqueza em outros trabalhos na região amazônica (Becker et al., 1991; Morato, 1994) e em Rebêlo e Garófalo (1997) na região Sudeste. Em outras regiōes, Sofia e Suzuki (2004), Neves e Viana (1997), Bezerra e Martins (2001), Brito e Rêgo (2001) também coletaram maior abundância e riqueza em eucaliptol, substância semelhante ao cineol. Nemésio (2003) e Peruquetti et al. (1999), ambos coletando no estado de Minas Gerais, não capturaram nenhum indivíduo em metil salicilato. Nemésio \& Morato (2004) verificaram maior atratividade de indivíduos e espécies em benzil acetato. Porém, isso pode estar relacionado ao fato de que esta isca foi a mais atrativa para as espécies dominantes (Nemésio e Morato, 2004).

Considerando o total de indivíduos coletados neste estudo, Eugenol foi a substância menos atrativa. Porém, atraiu maior número de indivíduos portando polinários. Peruquetti et al. (1999) coletaram machos de maior número de espécies em eugenol do que cineol.

Parâmetros climáticos podem interferir na volatilização dos compostos. Cineol, por exemplo, é muito mais volátil que eugenol e por isso tem uma melhor dispersão podendo atrair indivíduos de longas distâncias (Silva e Rebelo, 2002).

Além dos fatores climáticos a pureza e composição isomérica das substâncias (Williams e Whitten, 1983), a estrutura etária das populações (Zimmerman e Madrinam 1988; Ackerman, 1989; Rebêlo e Garófalo, 1997) e a oferta das substâncias por fontes naturais também podem interferir na atratividade das espécies.

Grande quantidade dos indivíduos, pertencentes a 29 espécies, foi coletada voando no local da coleta porém, sem coletar substância em uma isca odorífera. Dessa forma, a coleta desses indivíduos é importante para a estimativa da abundância de indivíduos de uma comunidade. É provável que ao movimentar a isca, moléculas de substâncias se espalhem na vegetação ou ainda que com a proximidade das iscas ocorra uma mistura de odores que confundem os machos.
Todos os indivíduos coletados portando polinários pertencem a espécies comuns (El. nigrita, El. cingulata, El. pseudocingulata, El. meriana, Eulaema bombiformis (Packard), 1869, El. mocsaryi, Euglossa ignita Smith, 1874, Eg. amazonica e Euglossa modestior Dressler, 1982). Esse fato sugere que essas abelhas estão realizando polinização nessas áreas. A grande maioria desses indivíduos foi coletada nos meses de abril, maio e junho, entre as 8:30 e 12:00 horas. É possível que estes sejam os períodos de maior oferta de substâncias pelas fontes naturais. Porém, a falta de conhecimento da flora local de Orchidaceae e de uma coleção palinológica para comparação dificulta as conclusōes sobre polinização. Peruquetti et al. (1999) coletaram 7 (0,58\%) machos pertencentes às espécies El. cingulata, Eg. amazonica e Eg. modestior, todas comuns no presente trabalho e também portando polinários. Neves e Viana (1997) coletaram 23 (2\%) machos pertencentes às espécies Euglossa cordata (Linnaeus), 1758, Eg. imperialis e El. nigrita. Pearson e Dressler (1985) coletaram no Peru $36(1,2 \%)$ indivíduos portando polinários pertencentes a 13 espécies, das quais 6 também foram coletadas portando polinários neste trabalho.

No Estado do Acre foram realizados poucos inventários, portanto, é possível que a riqueza de Euglossina seja muito maior na região, a exemplo de outros grupos de Aculeata (Azevedo et al., 2002; Souza et al., 2003; Morato e Martins, 2005). Em localidades da América Central, onde muitos estudos vêm sendo realizados, a riqueza de Euglossina é mais conhecida. Uma amostragem de cerca de seis dias consecutivos pode ser suficiente para amostrar a fauna local de Euglossina (Roubik e Hanson 2004). Porém, quanto maior for o esforço amostral, a separação temporal das coletas e a heterogeneidade de ambientes, maior será a probabilidade de se coletar espécies raras e, portanto maior riqueza.

A falta de um protocolo amostral padronizado tem dificultado a comparação dos trabalhos realizados em diferentes regiôes. Em virtude disso, alguns trabalhos (e.g. Ackerman, 1983) não puderam aqui ser comparados. A grande riqueza constatada no presente trabalho pode estar associada ao fato de que uma grande heterogeneidade espacial foi contemplada durante as amostragens. Portanto, um protocolo amostral de longo prazo, em áreas mais preservadas e heterogêneas, deve revelar no futuro a existência de espécies de Euglossina ainda não registradas na região.

\section{AGRADECIMENTOS}

Ao Programa de Pós-graduação em Ecologia e Manejo de Recursos Naturais da Universidade Federal do Acre.

Ao CNPq pela bolsa concedida.

Aos diretores e proprietários das áreas pela permissão da realização das amostragens. 
A Antonio Willian Flores de Melo pelo auxilio na escolha das áreas.

\section{BIBLIOGRAFIA CITADA}

Ackerman, J.D. 1983. Diversity and sasonality of male euglossine bees (Hymenoptera, Apidae) in Central Panama. Ecology, 64(2): 274-283.

Acre. 2006. Governo do Estado do Acre. Programa Estadual de Zoneamento Ecológico Econômico Fase II: documento Síntese Escala 1:250.000. SEMA, Rio Branco, Acre. 356p.

Anjos-Silva, E.J.dos; Camilo, E. \& Garófalo, C.A. 2006. Occurrence of Aglae caerulea Lepeletier \& Serville (Hymenoptera: Apidae: Euglossini) in the Parque Nacional da Chapada dos Guimarães, Mato Grosso State, Brazil. Neotropical Entomology, 35(6): 868870 .

Azevedo, C.O.; Helmer, J.L. \& Morato, E.F. 2002. Diversidade de Bethylidae (Hymenoptera) do Parque Nacional da Serra do Divisor, Acre, Brasil e seu uso no plano de manejo e conservação da área. Acta Amazonica, 32(1): 71-82.

Bawa, K.S. 1990. Plant-pollinators interactions in tropical rain forest. Annual Review of Ecology and Systematics, 21: 399-422.

Becker, P.; Moure, J.S. \& Peralta, F.J.A. 1991. More about Euglossine bees in Amazonian forest fragments. Biotropica, 23(4b): 586591.

Bezerra, C.P. \& Martins, C.F. 2001. Diversidade de Euglossinae (Hymenoptera. Apidae) em dois fragmentos de Mata Atlântica localizados na região urbana de João Pesssoa, Paraíba, Brasil. Revista Brasileira de Zoologia, 18(3): 823-835.

Braga, A.K. \& Garófalo, C.A. 2003. Coleta de fragrâncias por machos de Euglossa townsendi Cockerell (Hymenoptera, Apidae, Euglossini) em flores de Crinum procerum Carey (Amaryllidaceae). In: Melo, G.A.R. \& Alves-dos-Santos, I. (Eds). Apoidea Neotropica: Homenagem aos 90 anos de Jesus Santiago Moure. UNESC, Criciúma, Santa Catarina. p. 201-207.

Brito, C.M.S. de \& Rêgo, M.M.C. 2001. Community of male Euglossini bees (Hymenoptera: Apidae) in a secondary forest, Alcântara, MA, Braszil. Brazilian Journal of Biology, 61(4): 631-638.

Coleman, B.D.; Mares, M.A.; Willig, M.R. \& Hsieh, Y. 1982. Randomness, area and species richness. Ecology 63 (4):11211133.

Dodson, C.H.; Dressler, R.L.; Hills, H.G.; Adams, R.M. \& Williams, N.H. 1969. Biologically active compounds in orchid fragrances. Science, 164:1243-1249.

Dressler, R.L. 1982. Biology of the orchid bees (Euglossini). Annual Review of Ecology and Systematic, 13: 373-394.

Eltz, T.; Whitten, W.M.; Roubik, D.W. \& Linsenmair, K.E. 1999. Fragrance collection, storage, and accumulation by individual male orchid bees. Journal of Chemical Ecology, 25(1): 157-176.

Janzen, D.H.; DeVries, P.J.; Higgins, M.L. \& Kimsey, L.S. 1982. Seasonal and site variation in Costa Rican euglossine bees at chemical baits in lowland deciduous and evergreen forests. Ecology, 63(1): 66-74.
Magurran, A.E. 1988. Ecological Diversity and its Measurement. Princeton University Press. Princeton, NJ, USA. 179pp.

Martins, C.F. \& Souza, A.K.P. de. 2005. Estratificação vertical de abelhas Euglossina (Hymenoptera, Apidae) em uma área de Mata Atlântica, Paraíba, Brasil. Revista Brasileira de Zoologia, 22(4): 913-918

Minckley, R.L. \& Reyes, S.G. 1996. Capture of the orchid bee, Eulaema polychroma (Friese) (Apidae: Euglossini) in Arizona, with notes on northern distributions of other Mesoamerican bees. Journal of the Kansas Entomological Society, 69(1): 102104.

Milet-Pinheiro, P. \& Schlindwein, C. 2005. Do euglossine males (Apidae, Euglossini) leave tropical rain Forest to collect fragrances in sugarcane monocultures? Revista Brasileira de Zoologia, 22(4): 853-858.

Morato, E.F. 1994. Abundância e riqueza de machos de Euglossini (Hymenoptera: Apidae) em áreas de terra firme e áreas de derrubada, nas vizinhanças de Manaus (Brasil). Boletim do Museu Paraense Emilio Goeldi, Série Zoologia, 10(1): 95 -105.

Morato, E.F. 2001. Ocorrência de Aglae caerulea Lepeletier \& Serville (Hymenoptera, Apidae, Apini, Euglossina) no Estado do Acre, Brasil. Revista Brasileira de Zoologia 18, (3): 1031-10334.

Morato, E.F.; Campos, L.A. de O. \& Moure, J.S. 1992. Abelhas Euglossini (Hymenoptera, Apidae) coletadas na Amazônia Central. Revista Brasileira de Entomologia, 36(4): 767-771.

Morato, E.F. \& Martins, R.P. 2005. Diversidade e composição da fauna de vespas e abelhas solitárias do Estado do Acre, Amazônia Sul-Ocidental. In: Drumond, P.M. (Ed). Fauna do Acre. EDUFAC, Rio Branco, Acre. p.11-40.

Mori, S.A. \& Prance, G.T. 1987. Species diversity, phenology, plant-animal interactions, and their correlation with climate, as illustrated by Brazil nut family (Lecythidaceae), In: Dickinson, R.E. (Ed). The geographysiology of Amazonia. John Wiley \& Sons, New York, NY, USA. p. 68-69.

Nemésio, A. 2003. Preliminary sampling of Euglossina (Hymenoptera: Apidae: Apini) of Reserva Particular do Patrimônio Natural "Feliciano Miguel Abdala", Caratinga, Minas Gerais, southeastern Brazil. Lundiana, 4(2): 121-124.

Nemésio, A. 2005. Orchid bees (Hymenoptera: Apidae) of Ilha de Maracá, Roraima, northern Brazil. Lundiana, 6(2): 117-119.

Nemésio, A. \& Morato, E.F. 2004. Euglossina (Hymenoptera: Apidae) of the Humaitá Reserve, Acre state, Brazilian Amazon, with comments on bait trap efficiency. Revista de Tecnologia e Ambiente, 10(2): 71-80.

Nemésio, A. \& Morato, E.F. 2005. A diversidade de abelhas Euglossina (Hymenoptera: Apidae: Apini) do estado do Acre. In: Drumond, P.M (Ed). Fauna do Acre. EDUFAC, Rio Branco, Acre. p. 41-51.

Nemésio, A. \& Morato, E.F. 2006. The orchid-bee fauna (Hymenoptera: Apidae) of Acre state (northwestern Brazil) and a re-evaluation of euglossine bait-trapping. Lundiana, 7(1): 59-64.

Nemésio, A. \& Silveira, F.A. 2006. Edge Effects on the Orchid-Bee Fauna (Hymenoptera: Apidae) at a Large Remnant of Atlantic 
Rain Forest in Southeastern Brazil. Neotropical Entomology, 35(3): 313-323.

Neves, E.D. das \& Viana, B.F. 1997. Inventário da fauna de Euglossinae (Hymenoptera, Apidae) do baixo Sul da Bahia, Brasil. Revista Brasileira de Zoologia, 14(4): 831-837.

Neves, E.D. das \& Viana, B.F. 1999. Comunidade de machos de Euglossinae (Hymenoptera, Apidae) das matas ciliares da margem esquerda do médio rio São Francisco, Bahia. Anais da Sociedade Entomológica do Brasil, 28(2): 201-210.

Oliveira, M.L. 1999. Sazonalidade e horário de atividade de abelhas Euglossinae (Hymenoptera, Apidae), em florestas de terra firme na Amazônia Central. Revista Brasileira de Zoologia, 16(1): 83-90.

Oliveira, M.L. 2006. Três novas espécies de abelhas da Amazônia pertencentes ao gênero Eulaema (Hymenoptera: Apidae: Euglossini). Acta Amazônica, 36(1): 121-128.

Oliveira, M.L. \& Campos, L.A.O. 1995. Abundância, riqueza e diversidade de abelhas Euglossinae (Hymenopetera, Apidae) em florestas contínuas de terra firme na Amazônia Central, Brasil. Revista. Brasileira de Zoologia, 12(3): 547-556.

Oliveira, M.L. \& Campos, L.A.O. 1996. Preferências por estratos florestais e por substâncias odoríferas em abelhas Euglossinae (Hymenoptera, Apidae). Revista Brasileira de Zoologia, 13(4): 1075-1085.

Oliveira, M.L. \& Nemésio, A. 2003. Exaerete lepeletieri (Hymenoptera: Apidae: Apini: Euglossina): a new cleptoparasitic bee from Amazonia. Lundiana, 4(2): 117-120.

Otero, J.T. \& Sandino, J.C. 2003. Capture Rates of Male Euglossine Bees across a Human Intervention Gradient, Chocó Region, Colombia. Biotropica, 35(4): 520-529.

Pearson, D.L. \& Dressler, R.L. 1985. Two-year study of male orchid bee (Hymenoptera: Apidae: Euglossini) attraction to chemical baits in lowland south-eastern Peru. Journal of Tropical Ecology, 1: $37-54$.

Peruquetti, R.C.; Campos, L.A.O.; Coelho, C.D.P.; Abrantes, C.V.M. \& Lisboa, L.C.O. 1999. Abelhas Euglossini (Apidae) de áreas de Mata Atlântica: Abundância, riqueza e aspectos biológicos. Revista Brasileira de Zoologia, 16(Supl.2): 101-118.

Powell, A.H. \& Powell, G.V.N. 1987. Population Dynamics of Male Euglossine Bees in Amazonian Forest Fragments. Biotropica, 19(2): 176-179.

Prance, G.T. 1973. Phytogeographic support for the theory of Pleistocene Forest refuges in the Amazon basin based on evidence from distribution patterns in Caryocaraceae, Chrysobalanaceae, Dichapetalaceae and Leythidaceae. Acta Amazonica, 3(3): 5-28.

Rebêlo, J.M.M. \& Garófalo, C.A. 1997. Comunidades de machos de Euglossini (Hymenoptera: Apidae) em matas semidecíduas do nordeste do estado de São Paulo. Anais da Sociedade Entomológica do Brasil, 26(2): 243-255.

Rebêlo, J.M.M. \& Cabral, A. de J.M. 1997. Abelhas Euglossinae de Barreirinhas, Zona do Litoral da Baixada Oriental Maranhense. Acta Amazonica, 27(2): 145-152.
Roubik, D.W. \& Ackerman, J.D. 1987. Long-term ecology of euglossine orchid-bees (Apidae, Euglossini) in Panama. Oecologia, 73: 321-333.

Roubik, D.W. \& Hanson, P.E. 2004. Orchid bees of tropical America biology and field guide. INBio, Costa Rica, Panama. 370pp.

Silva, F.S. \& Rebelo, J.M.M. 2002. Population dynamics of Euglossinae bees (Hymenoptera, Apidae) in an early secondgrowth forest of Cajual Island, in the state of Maranhão, Brazil. Brazilian Journal of Biology, 62(1): 15-23.

Silveira, F.A.; Melo, G.A.R. \& Almeida, E.A.B. 2002. Abelhas brasileiras: sistemática e identificação. Ministério do Meio Ambiente, Fundação Araucária, Belo Horizonte, MG, Brasil. 253 pp.

Silveira, M. 2005. A floresta aberta com bambu no sudeste da Amazônia. Padrôes e processos em múltipla escala. EDUFAC, Rio Branco, Acre, Brasil. 145pp.

Skov, C. \& Wiley, J. 2005. Establishment of the neotropical orchid bee Euglossa viridissima (Hymenoptera: Apidae) in Florida. Florida Entomologist, 88(2): 225-227.

Soares, A.P.; Campos, L.A.O.; Vieira, M.F. \& Melo, G.A.R. de. 1989. Relaçôes entre Euglossa (Euglossella) mandibularis Friese, 1899 (Hymenoptera, Apidae, Euglossini) e Cyphomandra calycina (Solanaceae). Ciência e Cultura, 41(9): 903-905.

Sofia, S.H. \& Suzuki, K.M. 2004. Comunidades de Machos de Abelhas Euglossina (Hymenoptera: Apidae) em fragmentos Florestais no Sul do Brasil. Neotropical Entomology, 33(6): 693-702.

Sofia, S.H.; Santos, A. M. dos \& Silva, C.R.M da. 2004. Euglossine bees (Hymenoptera, Apidae) in a remnant of Atlantic Forest in Paraná State, Brazil. Iheringia Série Zoologia, 94(2): 217-222.

Sokal, R.R. \& Rohlf, F.J. 1995. Biometry. W. H. Freeman and Company, New York, NY, USA. 887pp.

Souza, A.K.P.; Hernández, M.I.M. \& Martins, C.F. 2005. Riqueza, abundância e diversidade de Euglossina (Hymenoptera, Apidae) em três áreas da Reserva Biológica Guaribas, Paraíba, Brasil. Revista Brasileira de Zoologia, 22(2): 320-325.

Souza, M.B. de; Silveira, M.; Lopes, M.R.M.; Vieira, L.J.S.; Guilherme, E.; Calouro, A.M. \& Morato, E.F. 2003. A biodiversidade no Estado do Acre: conhecimento atual, conservação e perspectivas. T \& C Amazônia, 1(3): 45-56.

Tonhasca, A.Jr.; Blackmer, J.L. \& Albuquerque, G.S. 2002. Abundance and Diversity of Euglossine Bees in the Fragmented Landscape of the Brazilian Atlantic Forest. Biotropica, 34(3): 416-422.

Williams, N.H. \& Whitten, W.M. 1983. Orchid floral fragrances and male euglossine bees: methods and advances in the last sesquidecade. Biological Bulletin, 164: 355-395.

Wittmann, D.; Hoffmann, M. \& Scholz, E. 1988. Southern distributional limits of euglossine bees in Brazil linked to habitats of the Atlantic and subtropical rain forest (Hymenoptera, Apidae, Euglossini). Entomologia Generalis, 14(2): 53-60.

Wittmann, D.; Radtke, R.; Hoffmann, M. \& Blochtein, B. 1989. Seasonality and seasonal changes in preferences for scent baits in 


\section{ACTA}

Euplusia violacea in Rio Grande do Sul- Brasil (Hymenoptera, Apidae, Euglossini). Entomologia Generalis, 14(3/4): 217-221.

Zimmerman, J.K. \& Madrinan, S.R. 1988. Age structure of male Euglossa imperialis (Hymenoptera, Apidae, Euglossini) at nectar and chemical sources in Panama. Journal of Tropical Ecology, 4: 303-306.

Recebido em 08/05/2008

Aceito em 22/05/2009 
